\title{
TOTALLY REAL THUE INEQUALITIES OVER IMAGINARY QUADRATIC FIELDS
}

\author{
István GaÁl, Borka JadRiJević And LÁsZló Remete
}

University of Debrecen, Hungary and University of Split, Croatia

\begin{abstract}
Let $F(x, y)$ be an irreducible binary form of degree $\geq 3$ with integer coefficients and with real roots. Let $M$ be an imaginary quadratic field with ring of integers $\mathbb{Z}_{M}$. Let $K>0$. We describe an efficient method how to reduce the resolution of the relative Thue inequalities

$$
|F(x, y)| \leq K \quad\left(x, y \in \mathbb{Z}_{M}\right)
$$

to the resolution of absolute Thue inequalities of type

$$
|F(x, y)| \leq k \quad(x, y \in \mathbb{Z}) .
$$

We illustrate our method with an explicit example.
\end{abstract}

\section{INTRODUCTION}

Let $F(x, y) \in \mathbb{Z}[x, y]$ be an irreducible binary form of degree $\geq 3$ and let $a \in \mathbb{Z} \backslash\{0\}$. There is an extensive literature of Thue equations of type

$$
F(x, y)=a \text { in } x, y \in \mathbb{Z} .
$$

In $1909 \mathrm{~A}$. Thue ([10]) proved that these equations admit only finitely many solutions. In 1967 A. Baker ([1]) gave effective upper bounds for the solutions. Later on authors constructed numerical methods to reduce the bounds and to explicitly calculate the solutions, see [6] for a summary.

2010 Mathematics Subject Classification. 11D59, $11 \mathrm{D} 57$.

Key words and phrases. Relative Thue equations, Thue inequalities.

Research of the first author is supported in part by K115479 from the Hungarian National Foundation for Scientific Research and by the EFOP-3.6.1-16-2016-00022 project. The project is co-financed by the European Union and the European Social Fund. Research of the second author is supported in part by the Croatian Science Foundation under the project no. 6422 . Research of the third author is supported by the ÚNKP-17-3 new national excellence program of the Ministry of human capacities. 
Let $M$ be an algebraic number field with ring of integers $\mathbb{Z}_{M}$. Let $F(x, y) \in \mathbb{Z}_{M}[x, y]$ be an irreducible binary form of degree $n \geq 3$ and let $\mu \in \mathbb{Z}_{M} \backslash\{0\}$. As a generalization of Thue equations consider relative Thue equations of type

$$
F(x, y)=\mu \text { in } x, y \in \mathbb{Z}_{M} .
$$

Using Baker's method S. V. Kotov and V. G. Sprindžuk ([8]) were first to give effective upper bounds for the solutions of relative Thue equations. Their theorem has been extended by several authors. Applying Baker's method, reduction and enumeration algorithms I. Gaál and M. Pohst ([7]) gave an efficient algorithm for solving relative Thue equations (see also [6]).

Let $M$ be an imaginary quadratic number field. Assuming in addition that the roots of $F(x, 1)$ are all real, in the present paper we give an efficient algorithm to reduce the resolution of relative Thue inequalities of the type

$$
|F(x, y)| \leq K \text { in } x, y \in \mathbb{Z}_{M}
$$

to the resolution of (absolute) Thue inequalities of the type

$$
|F(x, y)| \leq k \text { in } x, y \in \mathbb{Z} .
$$

To find the solutions of the above absolute Thue inequality one can use Kash ([4]) or Magma ([2]) which admit efficient algorithms for solving (absolute) Thue equations $F(x, y)=k^{\prime}$ for $k^{\prime} \in \mathbb{Z}$ with $\left|k^{\prime}\right| \leq k$. For an efficient method for calculating "small" solutions of Thue inequalities we refer to [9].

Our method is illustrated with an explicit example.

\section{The MAin Result}

Let $F(x, y)$ be a binary form of degree $n \geq 3$ with rational integer coefficients. Assume that $f(x)=F(x, 1)$ has leading coefficient 1 and distinct real roots $\alpha_{1}, \ldots, \alpha_{n}$. Let $0<\varepsilon<1,0<\eta<1$ and let $K \geq 1$. Set

$$
\begin{array}{cc}
A=\min _{i \neq j}\left|\alpha_{i}-\alpha_{j}\right|, & B=\min _{i} \prod_{j \neq i}\left|\alpha_{j}-\alpha_{i}\right|, \\
C=\max \left\{\frac{K}{(1-\varepsilon)^{n-1} B}, 1\right\}, & \\
C_{1}=\max \left\{\frac{K^{1 / n}}{\varepsilon A},(2 C)^{1 /(n-2)}\right\}, & C_{2}=\max \left\{\frac{K^{1 / n}}{\varepsilon A}, C^{1 /(n-2)}\right\}, \\
D=\left(\frac{K}{\eta(1-\varepsilon)^{n-1} A B}\right)^{1 / n}, & E=\frac{(1+\eta)^{n-1} K}{(1-\varepsilon)^{n-1}} .
\end{array}
$$

Let $m \geq 1$ be a squarefree positive integer, and set $M=\mathbb{Q}(i \sqrt{m})$. Consider the relative Thue inequality

$$
|F(x, y)| \leq K \text { in } x, y \in \mathbb{Z}_{M} .
$$


If $m \equiv 3(\bmod 4)$, then $x, y \in \mathbb{Z}_{M}$ can be written as

$$
\begin{gathered}
x=x_{1}+x_{2} \frac{1+i \sqrt{m}}{2}=\frac{\left(2 x_{1}+x_{2}\right)+x_{2} i \sqrt{m}}{2}, \\
y=y_{1}+y_{2} \frac{1+i \sqrt{m}}{2}=\frac{\left(2 y_{1}+y_{2}\right)+y_{2} i \sqrt{m}}{2}
\end{gathered}
$$

with $x_{1}, x_{2}, y_{1}, y_{2} \in \mathbb{Z}$.

If $m \equiv 1,2(\bmod 4)$, then $x, y \in \mathbb{Z}_{M}$ can be written as

$$
x=x_{1}+x_{2} i \sqrt{m}, y=y_{1}+y_{2} i \sqrt{m}
$$

with $x_{1}, x_{2}, y_{1}, y_{2} \in \mathbb{Z}$.

Theorem 2.1. Let $(x, y) \in \mathbb{Z}_{M}^{2}$ be a solution of (2.1). Assume that

$$
\begin{array}{ll}
|y|>C_{1} & \text { if } \quad m \equiv 3(\bmod 4), \\
|y|>C_{2} & \text { if } \quad m \equiv 1,2(\bmod 4) .
\end{array}
$$

Then

$$
x_{2} y_{1}=x_{1} y_{2} .
$$

I. Further, if $m \equiv 3(\bmod 4)$, then the following holds:

IA1. If $2 y_{1}+y_{2}=0$, then $2 x_{1}+x_{2}=0$ and

$$
\left|F\left(x_{2}, y_{2}\right)\right| \leq \frac{2^{n} K}{(\sqrt{m})^{n}} .
$$

IA2. If $\left|2 y_{1}+y_{2}\right| \geq 2 D$, then

$$
\left|F\left(2 x_{1}+x_{2}, 2 y_{1}+y_{2}\right)\right| \leq 2^{n} E .
$$

IB1. If $y_{2}=0$, then $x_{2}=0$ and

$$
\left|F\left(x_{1}, y_{1}\right)\right| \leq K \text {. }
$$

IB2. If $\left|y_{2}\right| \geq \frac{2}{\sqrt{m}} D$, then

$$
\left|F\left(x_{2}, y_{2}\right)\right| \leq \frac{2^{n}}{(\sqrt{m})^{n}} E .
$$

II. If $m \equiv 1,2(\bmod 4)$, then the following holds:

IIA1. If $y_{1}=0$, then $x_{1}=0$ and

$$
\left|F\left(x_{2}, y_{2}\right)\right| \leq \frac{K}{(\sqrt{m})^{n}} .
$$

IIA2. If $\left|y_{1}\right| \geq D$, then

$$
\left|F\left(x_{1}, y_{1}\right)\right| \leq E .
$$

IIB1. If $y_{2}=0$, then $x_{2}=0$ and

$$
\left|F\left(x_{1}, y_{1}\right)\right| \leq K .
$$


IIB2. If $\left|y_{2}\right| \geq \frac{D}{\sqrt{m}}$, then

$$
\left|F\left(x_{2}, y_{2}\right)\right| \leq \frac{E}{(\sqrt{m})^{n}} .
$$

Our result is a far reaching generalization of an idea of [5].

\section{Proof of the main Result}

In the proof of Theorem 2.1 we shall use the following Lemma.

Lemma 3.1. Let $x, y \in \mathbb{Z}, y \neq 0$. Assume that

$$
\left|\alpha_{i_{0}}-\frac{x}{y}\right| \leq \frac{d}{|y|^{n}}
$$

for some $i_{0}\left(1 \leq i_{0} \leq n\right)$ and $d>0$. If

$$
|y| \geq\left(\frac{d}{\eta A}\right)^{1 / n}
$$

then

$$
|F(x, y)| \leq d(1+\eta)^{n-1} \prod_{j \neq i_{0}}\left|\alpha_{j}-\alpha_{i_{0}}\right| .
$$

Proof. By our assumption, we have

$$
\left|\alpha_{j}-\frac{x}{y}\right| \leq\left|\alpha_{j}-\alpha_{i_{0}}\right|+\left|\alpha_{i_{0}}-\frac{x}{y}\right| \leq(1+\eta)\left|\alpha_{j}-\alpha_{i_{0}}\right|
$$

for $j \neq i_{0}$. Therefore

$$
\prod_{j=1}^{n}\left|\alpha_{j}-\frac{x}{y}\right|=\left|\alpha_{i_{0}}-\frac{x}{y}\right| \cdot \prod_{j \neq i_{0}}^{n}\left|\alpha_{j}-\frac{x}{y}\right| \leq \frac{d}{|y|^{n}} \cdot(1+\eta)^{n-1} \cdot \prod_{j \neq i_{0}}\left|\alpha_{j}-\alpha_{i_{0}}\right|,
$$

which implies our assertion.

Now we turn to the proof of our main result Theorem 2.1.

Proof. Let $(x, y) \in \mathbb{Z}_{M}^{2}$ be an arbitrary solution of (2.1) with $y \neq 0$. Let $\beta_{j}=x-\alpha_{j} y, j=1, \ldots, n$, then the inequality (2.1) can be written as

$$
\left|\beta_{1} \cdots \beta_{n}\right| \leq K \text {. }
$$

Let $i_{0}$ be the index with

$$
\left|\beta_{i_{0}}\right|=\min _{j}\left|\beta_{j}\right| .
$$

Then $\left|\beta_{i_{0}}\right| \leq K^{\frac{1}{n}}$ and together with (2.2) and (2.3) we get

$$
\left|\beta_{j}\right| \geq\left|\beta_{j}-\beta_{i_{0}}\right|-\left|\beta_{i_{0}}\right| \geq\left|\alpha_{j}-\alpha_{i_{0}}\right| \cdot|y|-K^{\frac{1}{n}} \geq(1-\varepsilon) \cdot\left|\alpha_{j}-\alpha_{i_{0}}\right| \cdot|y|
$$


for $j \neq i_{0}$. From the previous inequality and (3.1), we have

$$
\left|\beta_{i_{0}}\right| \leq \frac{K}{\prod_{j \neq i_{0}}\left|\beta_{j}\right|} \leq \frac{c}{|y|^{n-1}}
$$

with

$$
c=\frac{K}{(1-\varepsilon)^{n-1} \prod_{j \neq i_{0}}\left|\alpha_{j}-\alpha_{i_{0}}\right|} .
$$

By (3.2) we obtain

$$
\left|\alpha_{i_{0}}-\frac{x \bar{y}}{|y|^{2}}\right|=\left|\alpha_{i_{0}}-\frac{x}{y}\right| \leq \frac{c}{|y|^{n}},
$$

hence

which implies

$$
\left.\left|\alpha_{i_{0}}\right| y\right|^{2}-x \bar{y} \mid \leq \frac{c}{|y|^{n-2}}
$$

$$
|\operatorname{Im}(x \bar{y})| \leq \frac{c}{|y|^{n-2}} .
$$

Note that $\frac{c}{|y|^{n-2}}<\frac{1}{2}$ and $\frac{c}{|y|^{n-2}}<1$ for $m \equiv 3(\bmod 4)$ and $m \equiv 1,2(\bmod$ $4)$, respectively, according to (2.2) and (2.3). Therefore $|\operatorname{Im}(x \bar{y})|=\frac{1}{2} \mid x_{2} y_{1}-$ $x_{1} y_{2} \mid \sqrt{m}<\frac{1}{2}$ and $|\operatorname{Im}(x \bar{y})|=\left|x_{2} y_{1}-x_{1} y_{2}\right| \sqrt{m}<1$ for $m \equiv 3(\bmod 4)$ and $m \equiv 1,2(\bmod 4)$, respectively. Hence in both cases we have (2.4).

I. Let $m \equiv 3(\bmod 4)$.

IA. The inequality (3.2) implies $\left|\operatorname{Re}\left(\beta_{i_{0}}\right)\right| \leq \frac{c}{|y|^{n-1}}$, i.e.

$$
\left|\left(2 x_{1}+x_{2}\right)-\alpha_{i_{0}}\left(2 y_{1}+y_{2}\right)\right| \leq \frac{2 c}{|y|^{n-1}} .
$$

IA1. If $2 y_{1}+y_{2}=0$, then (3.3) yields $2 x_{1}+x_{2}=0$, and the inequality (2.1) has the form

$$
\left|F\left(\frac{x_{2} i \sqrt{m}}{2}, \frac{y_{2} i \sqrt{m}}{2}\right)\right| \leq K
$$

whence we get $(2.5)$.

IA2. If $2 y_{1}+y_{2} \neq 0$, then

$$
\begin{aligned}
\left|\left(2 x_{1}+x_{2}\right)-\alpha_{i_{0}}\left(2 y_{1}+y_{2}\right)\right| & \leq \frac{2 c}{|y|^{n-1}}=\frac{2 c}{\left|\frac{\left(2 y_{1}+y_{2}\right)+y_{2} i \sqrt{m}}{2}\right|^{n-1}} \\
& \leq \frac{2^{n} c}{\left|2 y_{1}+y_{2}\right|^{n-1}} .
\end{aligned}
$$

Since we have assumed

$$
\left|2 y_{1}+y_{2}\right| \geq\left(\frac{2^{n} c}{\eta A}\right)^{1 / n},
$$


Lemma 3.1 implies

$$
\left|F\left(2 x_{1}+x_{2}, 2 y_{1}+y_{2}\right)\right| \leq 2^{n} c(1+\eta)^{n-1} \prod_{j \neq i_{0}}\left|\alpha_{j}-\alpha_{i_{0}}\right|
$$

whence we get $(2.6)$.

IB. By the inequality $(3.2)$, we have $\left|\operatorname{Im}\left(\beta_{i_{0}}\right)\right| \leq \frac{c}{|y|^{n-1}}$, i.e.

$$
\sqrt{m}\left|x_{2}-\alpha_{i_{0}} y_{2}\right| \leq \frac{2 c}{|y|^{n-1}} .
$$

IB1. If $y_{2}=0$, then (3.4) implies $x_{2}=0$ and the inequality (2.1) has the form

whence we get $(2.7)$.

$$
\left|F\left(\frac{2 x_{1}}{2}, \frac{2 y_{1}}{2}\right)\right| \leq K
$$

IB2. If $y_{2} \neq 0$, then

$$
\left|x_{2}-\alpha_{i_{0}} y_{2}\right| \leq \frac{2 c}{\sqrt{m}|y|^{n-1}}=\frac{2 c}{\sqrt{m}\left|\frac{\left(2 y_{1}+y_{2}\right)+y_{2} i \sqrt{m}}{2}\right|^{n-1}} \leq \frac{2^{n} c}{(\sqrt{m})^{n}\left|y_{2}\right|^{n-1}} \text {. }
$$

Since

Lemma 3.1 implies

$$
\left|y_{2}\right| \geq\left(\frac{2^{n} c}{(\sqrt{m})^{n} \eta A}\right)^{1 / n}
$$

$$
\left|F\left(x_{2}, y_{2}\right)\right| \leq \frac{2^{n} c}{(\sqrt{m})^{n}}(1+\eta)^{n-1} \prod_{j \neq i_{0}}\left|\alpha_{j}-\alpha_{i_{0}}\right|
$$

which implies (2.8).

II. Let $m \equiv 1,2(\bmod 4)$.

IIA. The inequality (3.2) implies $\left|\operatorname{Re}\left(\beta_{i_{0}}\right)\right| \leq \frac{c}{|y|^{n-1}}$, i.e.

$$
\left|x_{1}-\alpha_{i_{0}} y_{1}\right| \leq \frac{c}{|y|^{n-1}} .
$$

IIA1. If $y_{1}=0$, then (3.5) yields $x_{1}=0$ and the inequality (2.1) has the form

$$
\left|F\left(i \sqrt{m} x_{2}, i \sqrt{m} y_{2}\right)\right| \leq K,
$$

whence we get $(2.9)$.

IIA2. If $y_{1} \neq 0$, then

$$
\left|x_{1}-\alpha_{i_{0}} y_{1}\right| \leq \frac{c}{|y|^{n-1}}=\frac{c}{\left|y_{1}+i \sqrt{m} y_{2}\right|^{n-1}} \leq \frac{c}{\left|y_{1}\right|^{n-1}} .
$$

Since we have assumed

$$
\left|y_{1}\right| \geq\left(\frac{c}{\eta A}\right)^{1 / n}
$$


Lemma 3.1 implies

$$
\left|F\left(x_{1}, y_{1}\right)\right| \leq c(1+\eta)^{n-1} \prod_{j \neq i_{0}}\left|\alpha_{j}-\alpha_{i_{0}}\right|
$$

whence we get $(2.10)$.

IIB. By the inequality (3.2) we have $\left|\operatorname{Im}\left(\beta_{i_{0}}\right)\right| \leq \frac{c}{|y|^{n-1}}$, i.e.

$$
\sqrt{m}\left|x_{2}-\alpha_{i_{0}} y_{2}\right| \leq \frac{c}{|y|^{n-1}} .
$$

IIB1. If $y_{2}=0$, then (3.6) implies $x_{2}=0$ and the inequality (2.1) has the form

$$
\left|F\left(x_{1}, y_{1}\right)\right| \leq K
$$

which is just our assertion (2.11).

IIB2. If $y_{2} \neq 0$, then

$$
\left|x_{2}-\alpha_{i_{0}} y_{2}\right| \leq \frac{c}{\sqrt{m}|y|^{n-1}}=\frac{c}{\left|y_{1}+i \sqrt{m} y_{2}\right|^{n-1}} \leq \frac{c}{(\sqrt{m})^{n}\left|y_{2}\right|^{n-1}} .
$$

Since

Lemma 3.1 implies

$$
\left|y_{2}\right| \geq\left(\frac{c}{(\sqrt{m})^{n} \eta A}\right)^{1 / n}
$$

$$
\left|F\left(x_{2}, y_{2}\right)\right| \leq \frac{c}{(\sqrt{m})^{n}}(1+\eta)^{n-1} \prod_{j \neq i_{0}}\left|\alpha_{j}-\alpha_{i_{0}}\right|
$$

whence we get (2.12).

\section{How to APply TheOREM 2.1}

In this section we give useful hints for a practical application of Theorem 2.1 .

Using the same notation let us consider again the relative Thue inequality (2.1). We describe our algorithm in the case I (for $m \equiv 3(\bmod 4))$ since the case II is completely similar.

1. If $|y| \leq C_{1}$ then we have only finitely many possible values for $y$ and hence for $y_{1}, y_{2}$, as well. For each possible $y$ and for all integers $\mu \in \mathbb{Z}_{M}$ with $|\mu| \leq K$ we calculate the roots of the equation $F(x, y)-\mu=0$ in

$x$. For such a root $x$ we calculate the corresponding $x_{1}, x_{2}$. If $x_{1}, x_{2}$ are integers, then $x \in \mathbb{Z}_{M}$ and $(x, y)$ is a solutions of $(2.1)$.

Alternatively, by $\left|\beta_{i_{0}}\right| \leq K^{\frac{1}{n}}$ we obtain $|x| \leq K^{\frac{1}{n}}+\max \left|\alpha_{j}\right| \cdot C_{1}$. We can simply enumerate and test the finitely many possible values of $x_{1}, x_{2}$ and $y_{1}, y_{2}$.

2. Assume that $|y|>C_{1}$.

(a) If $\left|2 y_{1}+y_{2}\right|<2 D$, then 
(i) If $\left|y_{2}\right|<2 D / \sqrt{m}$, then we have only finitely many values for $y_{1}, y_{2}$, we proceed as in 1 .

(ii) If $\left|y_{2}\right| \geq 2 D / \sqrt{m}$, then we use IB2. We solve $F\left(x_{2}, y_{2}\right)=k$ for all $k \in \mathbb{Z}$ with $|k| \leq 2^{n} E /(\sqrt{m})^{n}$. We determine the possible values of $y_{1}$ which satisfy $\left|2 y_{1}+y_{2}\right|<2 D$. We substitute $x_{2}, y_{1}, y_{2}$ into $x_{2} y_{1}=x_{1} y_{2}$ to see if there exist corresponding integer $x_{1}$.

(b) If $\left|2 y_{1}+y_{2}\right| \geq 2 D$, then we use IA2. We calculate the solutions $X=2 x_{1}+x_{2}, Y=2 y_{1}+y_{2}$ of $F(X, Y)=k$ for all $k \in \mathbb{Z}$ with $|k| \leq 2^{n} E$.

(i) If $\left|y_{2}\right|<2 D / \sqrt{m}$ then there are only finitely many possible values for $y_{2}$. We determine $y_{1}$ from $Y$. Using $X=2 x_{1}+x_{2}$ we set $x_{2}=X-2 x_{1}$, substitute $x_{2}=X-2 x_{1}, y_{1}, y_{2}$ into $x_{2} y_{1}=x_{1} y_{2}$ and test if there is a corresponding $x_{1}$ in $\mathbb{Z}$.

(ii) If $\left|y_{2}\right| \geq 2 D / \sqrt{m}$ we use IB2. We solve $F\left(x_{2}, y_{2}\right)=k$ for $|k| \leq 2^{n} E /(\sqrt{m})^{n}$. We determine $x_{1}, y_{1}$ from $x_{2}, y_{2}$ and $X, Y$.

For solving absolute Thue equations $F(x, y)=k$ for certain values $k \in \mathbb{Z}$ one can efficiently apply Kash ([4]) and Magma ([2]).

We remark that an appropriate choice of the parameters $\varepsilon, \eta$ of Thereom 2.1 makes the resolution much easier. It is worthy to keep $C_{1}, C_{2}$ and also $D$ small, to avoid extensive tests of small possible solutions. On the other hand, if $E$ is small, then there are fewer Thue equations (over $\mathbb{Z}$ ) to be solved. Of course we can not make all these constants simultaneously small, therefore we need to make a compromise, taking into consideration also the value of $K$ (which also determines the number of Thue equations to be solved). Usually it is worthy to try several values of $\varepsilon, \eta$ before we start solving (2.1).

\section{An EXAMPLE}

Let $M=\mathbb{Q}(i \sqrt{5})$, and let

$$
F(x, y)=x^{4}-9 x^{3} y-21 x^{2} y^{2}+88 x y^{3}+48 y^{4}
$$

and consider the solutions of

$$
|F(x, y)| \leq 20 \text { in } x, y \in \mathbb{Z}_{M} .
$$

The polynomial $F(x, y)$ is irreducible and the roots of $F(x, 1)$ are approximately

$$
-3.4271,-0.49938,2.7581,10.1684 \text {. }
$$

We may set $A=2.9278, B=101.7426$. Further, let $\varepsilon=0.1$ and $\eta=0.1$. We are in case II. Calculating the constants, Theorem 2.1 gives:

Assume $|y|>7.2229$. Then:

IIA1. If $y_{1}=0$, then $x_{1}=0$ and $\left|F\left(x_{2}, y_{2}\right)\right| \leq 0.8000$.

IIA2. If $\left|y_{1}\right| \geq 0.9796$, then $\left|F\left(x_{1}, y_{1}\right)\right| \leq 36.5157$. 
IIB1. If $y_{2}=0$, then $x_{2}=0$ and $\left|F\left(x_{1}, y_{1}\right)\right| \leq 20$.

IIB2. If $\left|y_{2}\right| \geq 0.4381$, then $\left|F\left(x_{2}, y_{2}\right)\right| \leq 1.4606$.

First we consider the values with $|y| \leq C_{2}=7.2229$. We have $|x| \leq$ $20^{\frac{1}{4}}+\max \left|\alpha_{j}\right| \cdot C_{2}=75.64$. Enumerating and testing all possible $x=x_{1}+$ $i \sqrt{5} x_{2}$ and $y=y_{1}+i \sqrt{5} y_{2}$ satisfying these bounds we obtain the solutions $\left(x_{1}, x_{2}, y_{1}, y_{2}\right)=(0,0,0,0),(1,0,0,0),(2,0,0,0),(1,0,-2,0),(2,0,-4,0)$, up to sign.

If $y_{1}=0$ then by IIA 1 we have $x_{1}=0$ and $\left|F\left(x_{2}, y_{2}\right)\right| \leq 0.8$, whence $\left|F\left(x_{2}, y_{2}\right)\right|=0, x_{2}=0, y_{2}=0$.

If $y_{2}=0$ then by IIB1 we have $x_{2}=0$ and $\left|F\left(x_{1}, y_{1}\right)\right| \leq 20$. Using Magma we solve $F\left(x_{1}, y_{1}\right)=k$ for $-20 \leq k \leq 20$. We obtain the solutions $\left(x_{1}, y_{1}\right)=(0,0),(1,0),(1,-2),(2,0),(2,-4)$, up to sign. These bring the above solutions $\left(x_{1}, x_{2}, y_{1}, y_{2}\right)$ again.

From now on we assume that $y_{1} \neq 0$ and $y_{2} \neq 0$.

If $\left|y_{1}\right| \leq 0.9796$ and $\left|y_{2}\right| \leq 0.4381$ then by IIA2 we have $\left|F\left(x_{1}, y_{1}\right)\right| \leq$ 36.5157 and by IIB2 we have $\left|F\left(x_{2}, y_{2}\right)\right| \leq 1.4606$. In addition to the above calculation we solve $F\left(x_{1}, y_{1}\right)=k$ for $21 \leq|k| \leq 36$ but we do not get any further solutions. Hence the solutions of $\left|F\left(x_{1}, y_{1}\right)\right| \leq 36.5157$ are $\left(x_{1}, y_{1}\right)=(0,0),(1,0),(1,-2),(2,0),(2,-4)$, up to sign. Also the solutions of $\left|F\left(x_{2}, y_{2}\right)\right| \leq 1.4606$ are $\left(x_{1}, y_{1}\right)=(0,0),(1,0),(1,-2)$, up to sign. Testing these possible $\left(x_{1}, x_{2}, y_{1}, y_{2}\right)$ we do not get any new solutions.

If either $\left|y_{1}\right|<0.9796$ or $\left|y_{2}\right|<0.4381$ then $y_{1}=0$ or $y_{2}=0$ which cases we have already considered.

Hence all solutions of $(5.1)$ are $(x, y)=(0,0),(1,0),(2,0),(1,-2),(2,-4)$, up to sign. The calculation takes just a few seconds.

\section{REFERENCES}

[1] A. Baker, Transcendental number theory, Cambridge University Press, Cambridge, 1990.

[2] W. Bosma, J. Cannon and C. Playoust, The Magma algebra system.I. The user language, J. Symbolic Comput. 24 (1997), 235-265.

[3] B. W. Char, K. O. Geddes, G. H. Gonnet, M. B. Monagan, S. M. Watt, MAPLE, Reference Manual, Watcom Publications, Waterloo, Canada, 1988.

[4] M. Daberkow, C. Fieker, J. Klüners, M. Pohst, K.Roegner and K. Wildanger, KANT V4, Computational algebra and number theory, J. Symbolic Comput. 24 (1997), 267283. http://www . math.tuberlin.de/ kant/.

[5] I. Gaál, Computing elements of given index in totally complex cyclic sextic fields, J. Symbolic Comput. 20 (1995), 61-69.

[6] I. Gaál, Diophantine equations and power integral bases, Birkhäuser, Boston, 2002.

[7] I. Gaál and M. Pohst, On the resolution of relative Thue equations, Math. Comput. 71 (2002), 429-440.

[8] S. V. Kotov and V. G. Sprindžuk, An effective analysis of the Thue-Mahler equation in relative fields (Russian), Dokl. Akad. Nauk. BSSR 17 (1973), 393-395, 477.

[9] A. Pethő, On the resolution of Thue inequalities, J. Symbolic Comput. 4 (1987), 103109. 
[10] A. Thue, Über Annäherungswerte algebraischer Zahlen, J. Reine Angew. Math. 135 (1909), 284-305.

I. Gaál

University of Debrecen

Mathematical Institute

H-4002 Debrecen Pf.400.

Hungary

E-mail: gaal.istvan@unideb.hu

B. Jadrijević

University of Split

Faculty of Science

Ruđera Boškovića 33, 21000 Split

Croatia

E-mail: borka@pmfst.hr

L. Remete

University of Debrecen

Mathematical Institute

H-4002 Debrecen Pf.400.

Hungary

E-mail: remete.laszlo@science.unideb.hu

Received: 15.1.2018.

Revised: 2.5.2018. 